\title{
Fonctionnement hydrologiquede la grande mare de Kokorou dans le socle cristallin du Liptako Gourma (Niger)
}

\author{
Abdoulaye BABA ${ }^{1 *}$, Oumarou Faran MAÏGA ${ }^{1}$, Boureima OUSMANE ${ }^{2}$, Da Dapola \\ Evariste CONSTANT ${ }^{3}$ et Guillaume FAVREAU ${ }^{4}$
}

\author{
${ }^{1}$ Département de Géographie, Université Abdou Moumouni de Niamey, BP 418, Niger. \\ ${ }^{2}$ Département de Géologie, Université Abdou Moumouni de Niamey, BP 10996, Niger. \\ ${ }^{3}$ Département de Géographie, Université Ouaga 1 Pr Joseph Ki-Zerbo, Ouagadougoudou 03 BP 7021 , \\ Burkina Faso. \\ ${ }^{4}$ Institut de Recherche pour le Développement, UMR HydroSciences Montpellier (HSM), 5045, F-34032, \\ France. \\ *Auteur correspondant ; E-mail : abdoulayebaba@ymail.com ; Tél : +(227) 96062143
}

\section{RESUME}

La modification du fonctionnement hydrologique et ses effets sur le cycle de l'eau constituent une des conséquences majeures des changements environnementaux au Sahel. La mare de Kokorou, le plus grand plan d'eau naturel de l'Ouest nigérien, appartient à cette zone sahélienne de socle. Elle varie en superficie de 520 à 2398 ha en saison sèche et humide (période 1997 - 2017) et conjugue les différentes contraintes d'un usage raisonné de la ressource en eau de surface et des services rendus écosystémiques. La présente étude a pour objectif de décrire le fonctionnement hydrologique de cettegrande mare sahélienne permanente. Pour ce faire, les variations limnimétriques et surfaciques sont suivies et analysées à une échelle saisonnière et interannuelle. Il ressort que depuis près d'une dizaine d'années, la mare est permanente, occasionnant des mutations écologiques. L'accroissement des apports hydriquesdû à la dégradation du bassin versant et la baisse de l'infiltration au niveau du plan d'eau sont à l'origine de la modification du régime de la mare.

(C) 2018 International Formulae Group. All rights reserved.

Mots clés : Socle cristallin, mare de Kokorou, Liptako Gourma, remplissage, vidange.

\section{Hydrology of the large pool of Kokorou in the crystalline basement of Liptako Gourma (Niger)}

\begin{abstract}
The modification of hydrology and its effects on the water cycle is one of the main environmental consequences. Kokorou's pond, the largest pond of the western part of Niger belongs to this sahelian basement. It varies in area between 520 to 2398 hectares in dry and humid season (period of 1997 to 2017) and combines the different constraints of a sustainable using of the water body and the ecosystem services. This study aimed at describing the hydrological functioning of this permanent sahelian large pool. The limnimetric and surface variations are monitored and analyzed at the seasonal and annual scales. It appears that for nearly 10 years, the pond has become permanent causing ecological changes. The flows increasing due to the degradation of the watershed and the infiltration decreasing in the pond are responsible of the hydrological regime modification. (C) 2018 International Formulae Group. All rights reserved.
\end{abstract}

Keywords : Crystalline basement, Liptako Gourma, pool of Kokorou, recharge, emptying. 


\section{INTRODUCTION}

En milieu sahélien, les retenues d'eau de surface occupent une place importante notamment en zone de socle cristallin où la nappe est particulièrement profonde et souvent contaminée (Abdou Babaye et al., 2016). En plus d'être le lieu de pâturage, elles sont le lieu de prélèvement d'eau pour les besoins domestiques. C'est également le lieu de maraîchage et de pêche àl'exemple duLac Tchad (Kiari, 2014). Cette importance devient capitale au cours de la longue saison au moment où les petites mares se sont asséchées. Elles sont, en outre, fortement sensibles aux changements climatiques et environnementaux qui modifient leur fonctionnement hydrologique (Lemoalle, 2004; Leblanc et al., 2008; Ali et Lebel, 2009 ; Favreau, et al., 2009) voire même leur bilan hydrique. A cet effet, la diminution de $10 \%$ de la pluie sur le bassin tchadien entraîne, en effet, une diminution de $30 \%$ des apports au Lac Tchad par le Chari (Lemoalle, 2004) accompagnée d'une modification des paysages autour du lac (Lemoalle, 2010; Kiari, 2014).

Par ailleurs, à la suite des sécheressessévères et récurrentes et despériodes de famineassociées des années 1970-1980, l'Etat du Niger s'est tourné vers lamise en valeur de ces ressources en eau de surface pourl'atteinte de l'autosuffisance alimentaire au regard des faibles performances en période de sécheresse de l'agriculture pluviale. La première étape du projet a consisté à répertorier toutes les mares et à équiper un certain nombre d'entre elles en dispositifs de suivi. La mare de Kokorou, du fait de son importante superficie, fait partie des quelques mares du Liptako Gourma ayant bénéficié de ce projet. L'étude du fonctionnement de ces retenues en complétant les travaux hydrogéologiques existants (Ousmane et al., 2010 ; Abdou Babaye, 2012) pourra ainsi permettre de mieux appréhender la complexité de l'hydrologie de la zone.

L'objectif principal de cette étudeest de proposer un modèle de fonctionnement de la mare de Kokorou.

\section{MATERIEL ET METHODES Zone d'étude}

Située en zone de socle cristallin du Liptako Gourma (Figure 1) dansle Sud-ouest nigérien, la mare de Kokorou est le lieu de refuge pour les oiseaux migrateurs, mais c'est surtout le lieu de pâturage et d'abreuvage pour le bétail de la région attiré par son important potentiel en ressources fourragères et sa ressource hydrique intarissable. Le plan d'eau concentre les eaux drainées par cinq principaux collecteurs et celles directement tombées sur la mare. Son bassin versant est d'une superficie de $217 \mathrm{~km}^{2}$. La mare connaît actuellement d'importants changements écologiques liés à son fonctionnement hydrologique associée aux activités agricoles et halieutiques.

\section{Limnimétrie de la mare}

La collecte des données limnimétriques s'effectue suivant un réseau d'échelles qui s'emboîtent.Ces échelles, au nombre de quatre ont été installéespar la DREE, (DRRE/TI, 2001). Sur les quatre, les échelles $\mathrm{n}^{\circ} 3$, (position $14^{\circ} 12,241^{\prime} \mathrm{N}$ et $0^{\circ} 55,697^{\prime} \mathrm{E}$ ) et $\mathrm{n}^{\circ} 4$ (position $14^{\circ} 12,271^{\prime} \mathrm{N}$ et $0^{\circ} 55,705^{\prime} \mathrm{E}$ ) sont le plus souvent exondées.Le suivi journalier des échelles donne un niveau statique observé le matin et un niveau dynamique observé au coucher du soleil. Les variations journalières de la hauteur sont suivies (toutes les 12 heures) par lecture directe des échelles limnimétriques. L'observation des échelles a permis de faire un suivi synchronisé des différents paramètres du fonctionnement de la mare (hauteur et surface) à partir de chronique des années suivantes: 1997, 2005, 2006, 2007, 2008, 2010, 2011,2012, 2013 2014, 2015, 2016 et 2017.L'analyse du fonctionnement de la mare porte sur cette chronique.

\section{Plan d'eau}

L'objectif de la mesure de la surface est d'établir le rapport Hauteur/Surface de la mare. Pour ce faire, les différentes surfaces ont étémesurées et synchronisées aux niveaux limnimétriques observés. Ainsi, 28points de 
corrélation limnimétrique, à variation irrégulière allant de 10 à $15 \mathrm{~cm}$ ont été retenus. Les valeurs limnimétriques situées entre ces points sont déterminées par interpolation à l'aide de la méthode d'ajustement polynomial (polynôme d'ordre 3) de même que pour les surfaces correspondantes.Pour chaque point, la surface correspondante est mesurée sous Arc gis 10.4 par digitalisation des limites du plan d'eau. La détermination des limites du plan d'eau s'est faite à travers trois méthodes complémentaires. a.) La première a concerné les surfaces correspondantes aux niveaux 1,42 $\mathrm{m}, 1,02 \mathrm{~m}, 0,92 \mathrm{~m}$, et 0,82 , ayant été levées topographiquement par la Direction Régionale des Ressources en Eau de Tillabéri (DRRE/TI, 2001). Les cartes issues des levés ont été scannées, géoréférencées et numérisées pour lecalcul des surfaces. b.) La deuxième méthode a consisté à enun suivi planimétrique de lalimite du plan d'eau lors de la mission du 27 mars 2014 correspondant au niveau limnimétrique 2,34 m. Les points GPX collectés ont été exportés dans Arc gis puis reliés par numérisation pour le calcul de la superficie. c.) Enfin, les surfaces correspondantes aux niveaux restantsont été mesurées par numérisation d'images Landsat. A chacun de ces derniers niveaux limnimétriques, une image correspondant à la même datea été récupérée sur le sitehttps://earthexplorer.usgs.gov/ de l'United States Geological Survey (USGS). Au total, 23 scènes d'images Landsat MSS et ETM+ ont été numérisées. Pour toutes les images, la composition colorée retenue pour la numérisation comporteune bande de l'Infrarouge, une bande de l'Infrarouge thermique et une bande du Visible. Cette composition est idéale pour bien discriminer l'eau libre visà vis de l'eau boueuse et les limites de l'eau libre couverte de végétation aquatique d'avec les limites de la végétation terrestre et des cultures irriguées.

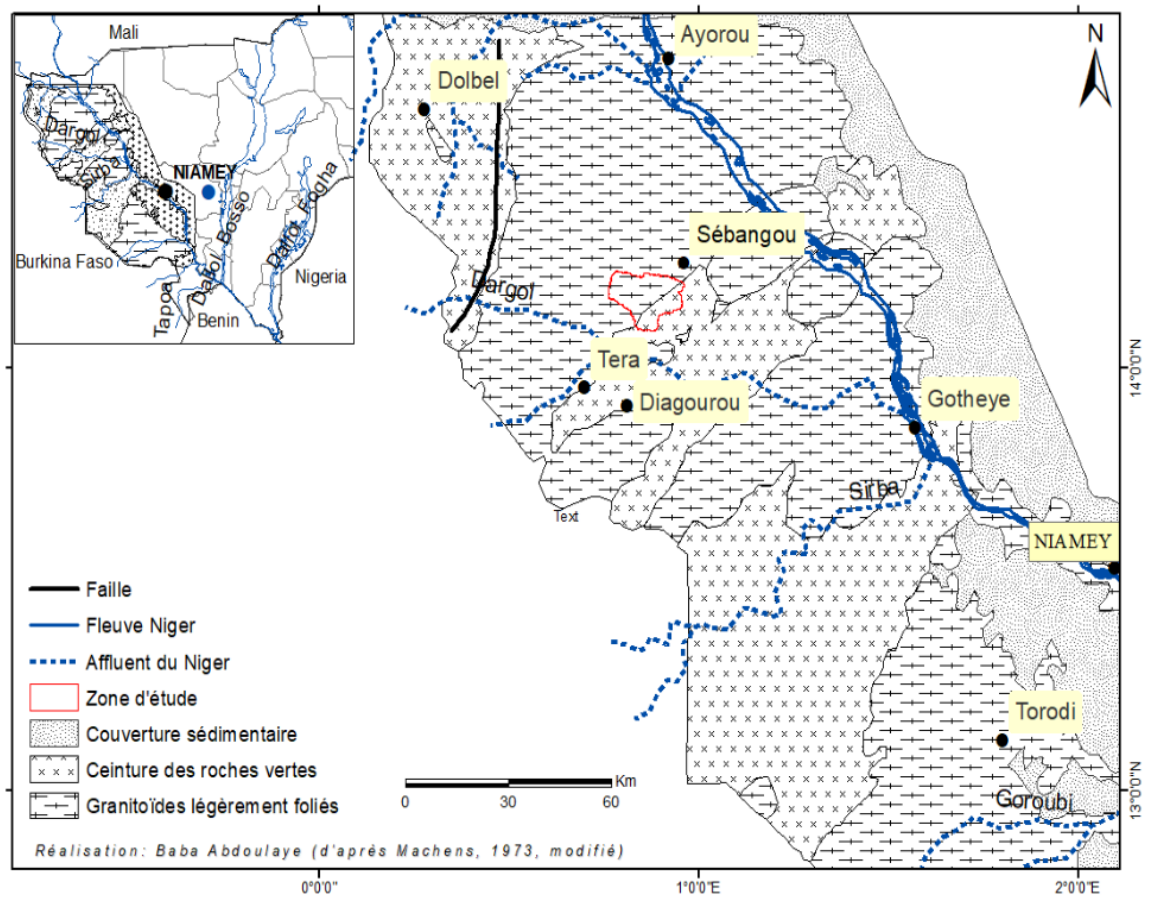

Figure 1 : Carte géologique de la région de socle du Liptako et de localisation du bassin versant de la mare de Kokorou au SO du Niger. 


\section{RESULTATS}

Le fonctionnement hydrologique de la mare de Kokorou comporte une courte phase de remplissage qui a lieu pendant la saison des pluies, entre juin et septembre et une longue phase de vidange correspondant à la saison sèche. Son cycle hydrologique est marqué par une variation saisonnièreconcomitantedes différents paramètres de son fonctionnement (hauteur, surface et volume du plan d'eau). Elle connaît ses hautes eaux entreseptembre et octobre et son étiage intervient en juin avant les premières pluies. Sa durée varie entre 8 à 12 mois selon toujours la saison des pluies qui est son principal facteur de remplissage.

Il existe une étroite interrelation entre les différents paramètres du fonctionnement (Figure 2). La hauteur du plan d'eau varie suivant les paramètres hydro-climatiques et humains à savoir le début de la saison des pluies, les hauteurs de pluies enregistrées et leur répartition dans le temps, le niveau initial (niveau de la cote avant le début de la recharge), l'évaporation et la consommation.

Le début de la recharge est très aléatoire.Il intervient entre les mois de juin et juillet avec des décalages entre les années. L'écart interannuel sur la période de suivi atteint 50 jours entre 1996 (où elle a débuté le 09 juin) et 2006 (où elle a débuté le 29 juillet).

La date du début de la recharge est importante car elle détermine un autre facteur déterminant pour le bilan hydrique annuel, à savoir le niveau initial de la cote. Ce niveau initial est aussi très variable et présente également des écarts significatifs. Le niveau initial maximal peut atteindre $2,01 \mathrm{~m}$, enregistré en 2017 ; le plus bas est de $0,41 \mathrm{~m}$ enregistré en 2008 où la pluviométrie annuelle a été de $475 \mathrm{~mm}$. L'écart entre ces deux années extrêmes est de 1,47 m. Notons que ce niveau initial dépend aussi de l'effet mémoire de la recharge pendant la saison de pluies précédente, il se répercute en retour sur la recharge comme c'est le cas entre 2006 et 2007 où la recharge débute avec des niveaux $\mathrm{N}_{0}$ respectifs de $0,54 \mathrm{~m}$ et $0,62 \mathrm{~m}$ (Figure 3).
Un épisode de recharge arrive à terme à partir du moment où la cote entame une baisse irréversible jusqu'à une prochaine phase de recharge, cette date est donc celle de la fin de la recharge, elle permet de déterminer, selon la date du début, le nombre de jours total qu'a duré la phase de recharge conséquemment à la durée de la saison de pluies. Cette durée de la recharge varie fortement, allant de 42 jours en 1997 à 111 jours en 2007.Elle influence l'allure de la courbe de recharge, en ce sens que, plus la durée est longue plus la courbe de recharge est linéaire, quand le temps de la recharge est bref, la courbe présente plus de fluctuations (Figure 4). Cependant, l'élément le plus important du temps de la recharge est de permettre la détermination d'une intensité moyenne de recharge $(H)$. L'intensité moyenne de la recharge est obtenue en faisant le rapport du dénivelé (entre le niveau avant le début et celui à la fin de la recharge) sur la durée de la recharge. C'est-à-dire:

$H=\frac{\mathrm{N} m-\mathrm{N} 0}{D}$

Avec pour paramètres de l'équation:

$H$ : Intensité moyenne de recharge [L]

$\mathrm{N}_{m}$ : Niveau maximal atteint à la fin de la recharge [L]

$\mathrm{N}_{0}$ : Niveau initial de la recharge [L]

$D$ : Durée de la recharge [T]

L'intensité moyenne de la recharge obtenue sur les années d'observation est de $2,64 \mathrm{~cm} /$ jour. Cette valeur masque cependant de fortes disparités interannuelles liées à la variabilité de tous les autres paramètres. Ainsi, l'intensité la plus élevée est enregistrée en 2008 avec une intensité de $5,07 \mathrm{~cm} /$ jour et la plus faible est de $0,66 \mathrm{~cm} /$ jour enregistrée en 2007. Ces deux annéescaractéristiques présentent inversement aux intensités moyennes des niveaux maximaux $(\mathrm{N} m)$ faibles par rapport aux années 2010 et 2014, 2016 (respectivement $3,05,3,15$ et $3,7 \mathrm{~m}$ ). Il existe alors une relation inverse entre l'intensité moyenne et la durée de la recharge telle que vérifiable sur l'équation: $H$ varie 
inversement à $D$. Cela se vérifie, de façon pratique, par le fait que plus le temps d'exposition du plan d'eau pendant la recharge $(D)$ est long, plus l'évaporation est importante et donc la valeur de $H$ baisse.

Les interrelations que nous venons de voir concernent, à priori, les autres paramètres du fonctionnement de la mare, à savoir la surface et le volume. Ainsi, l'analyse des chroniques limnimétriques combinée à l'exploitation d'images Landsat MSS et ETM+montre que la mare de Kokorou est passée de la situation d'une mare semipermanente à permanente à une mare totalement permanente (Figure 4). En effet, depuis la sécheresse de 2009, la mare n'a pas tari. Et depuis l'année 2010, le niveau limnimétrique à la fin de la phase de recharge atteint et dépasse le niveau $3 \mathrm{~m}$ alors qu'avant cette année, ce niveau n'était qu'exceptionnellement atteint (Figure 5).

Néanmoins, le nombre de crues varie faiblement à l'échelle interannuelle par rapport aux autres caractéristiques et est même en légère baisse depuis 2012 (Figure 6 a). La hausse des amplitudes en fin de recharge associée à l'élévation du niveau initial $\left(N_{0}\right)$ en début de recharge et du niveau maximal en fin de recharge $\left(N_{m}\right)$ sont les signes des changements qui affectent le bilan hydrique de la mare. Ce changement majeur de la mare de Kokorou est corroboré par les résultats d'une enquête auprès des populations riveraines. En effet, 95\% des personnes enquêtées reconnaissent non seulement que la mare ne tarit plus mais aussi qu'elle est en extension. Il ressort, pour ces populations, que le changement est tel que la superficie actuelle de la mare en période de basses eaux correspond globalement à celle des hautes eaux d'il y a neuf à dix ans tel que le montre la Figure 4.

La modification du fonctionnement hydrologique de la mare suivie de l'accroissement de son potentiel hydrique s'explique par un accroissement des apports vers la mare combinée à la modification probable des termes de son bilan.
Relativement à l'accroissement des apports vers le plan d'eau, il est à noter que d'une part, le bassin versant de la mare connaît actuellement une amélioration pluviométrique à l'instar de la zone sahélienne. En effet, la décennie qui commence à partir de 2010 pourrait être la plus pluvieuse (Figure 7) avec une moyenne actuelle (2010-2017) de 493 mm contre une moyenne décennale de 394 $\mathrm{mm}$ entre 2000 et 2009 et $427,5 \mathrm{~mm}$ pour la décennie 1990-1999 qui est déjà considérée comme le début d'une remontée pluviométrique au Sahel. La modification pluviométrique a un impact significatif sur la quantité d'eau directement tombée sur le plan d'eau est sur les hauteurs d'eau ruissélées. D'autre part, les changements importants de l'occupation des sols qui ont lieu dans le bassin versant de la mare, considérée par 51\% de la population enquêtée comme résultant de l'installation du site orifère de Komabangou en 1984 situé à $15 \mathrm{~km}$ du bassin versant,ont considérablement augmenté les ruissellements. Le bassin versant de la mare étant le lieu de prélèvement de bois d'œuvre pour les activités du site et pour la construction des habitations. Enfin, jusqu'en 2009, la mare de Kokorou était restée une mare très filtrante du fait de sa structure géologique, marquée par la présence de failles dans son lit qui favorisaient l'infiltration (Baba, 2012). Ces zones à écoulement inferroflux dont deux étaient remarquables en période de basses eaux (Communication orale) pourraient avoir été définitivement colmatées par l'importance des particules argileuses et limoneuses, provenant de la dégradation du bassin versant, qui se déposent pendant toute l'année mais aussi par le piétinement accentué du bétail à la suite de la dernière sécheresse. Il est à noter que le lit de la mare est le lieu de pâturage en période de basses eaux quand celle-ci est totalement (ou presque) à sec. Ce qui était le cas de la mare pendant l'étiage de 2009, le bétail avait donc accès à l'ensemble du lit. Enfin, la deuxième hypothèse serait la tendance à la saturation de l'aquifère de fracture de roche. 


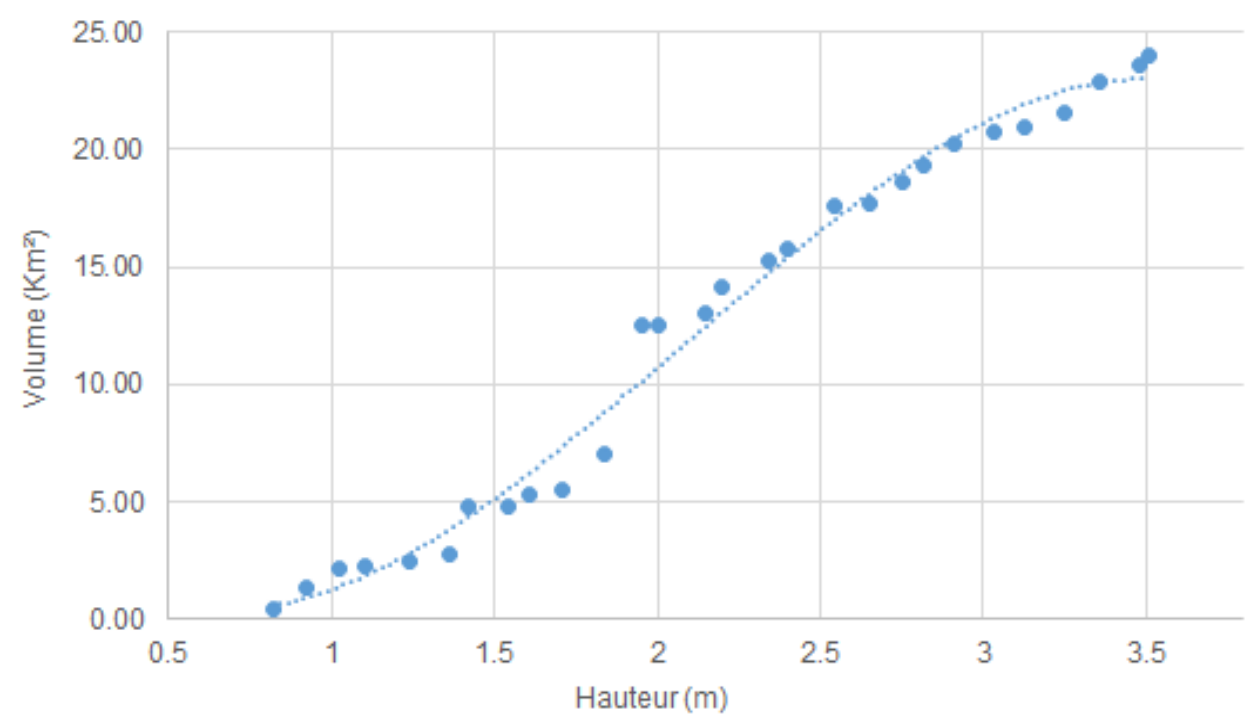

Figure 2 : Relation entre la hauteur et la surface. Source : (DRRE/TI).

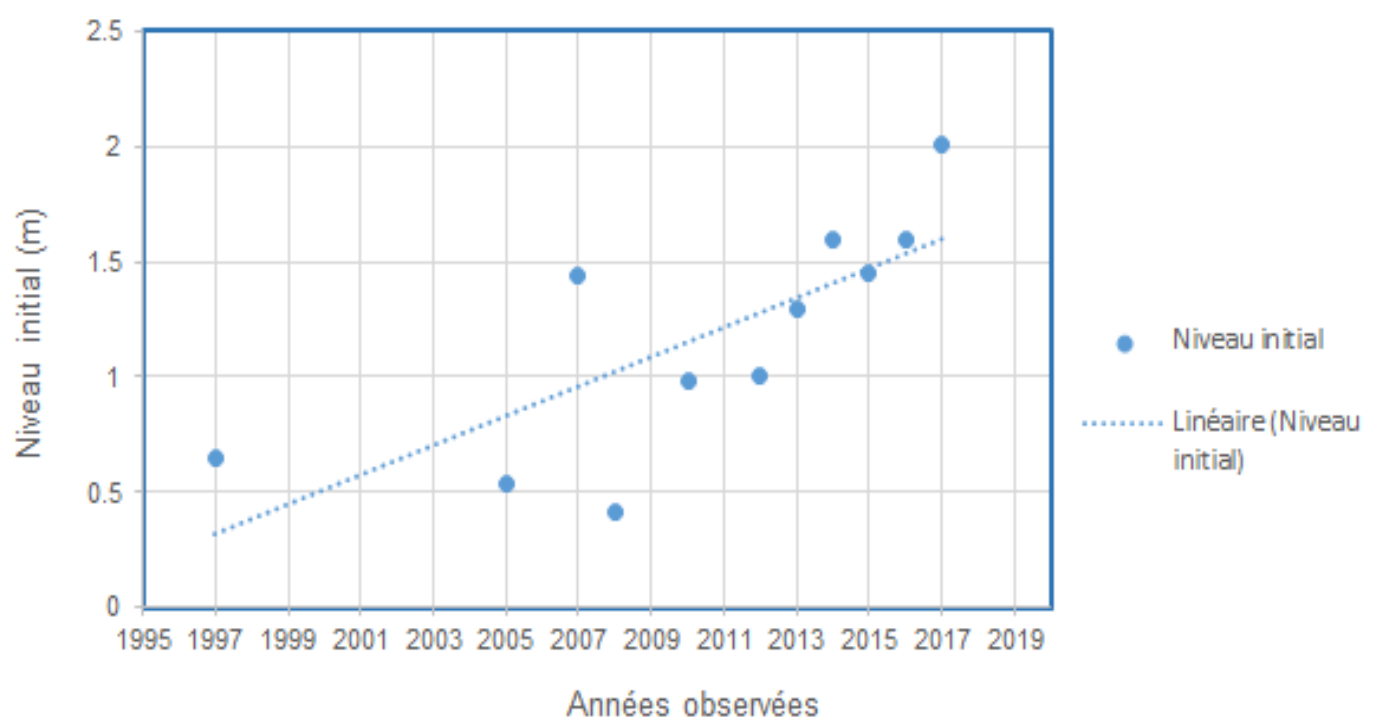

Figure 3 : Variation interannuelle du niveau initial de la recharge. Source : (DRRE/TI). 


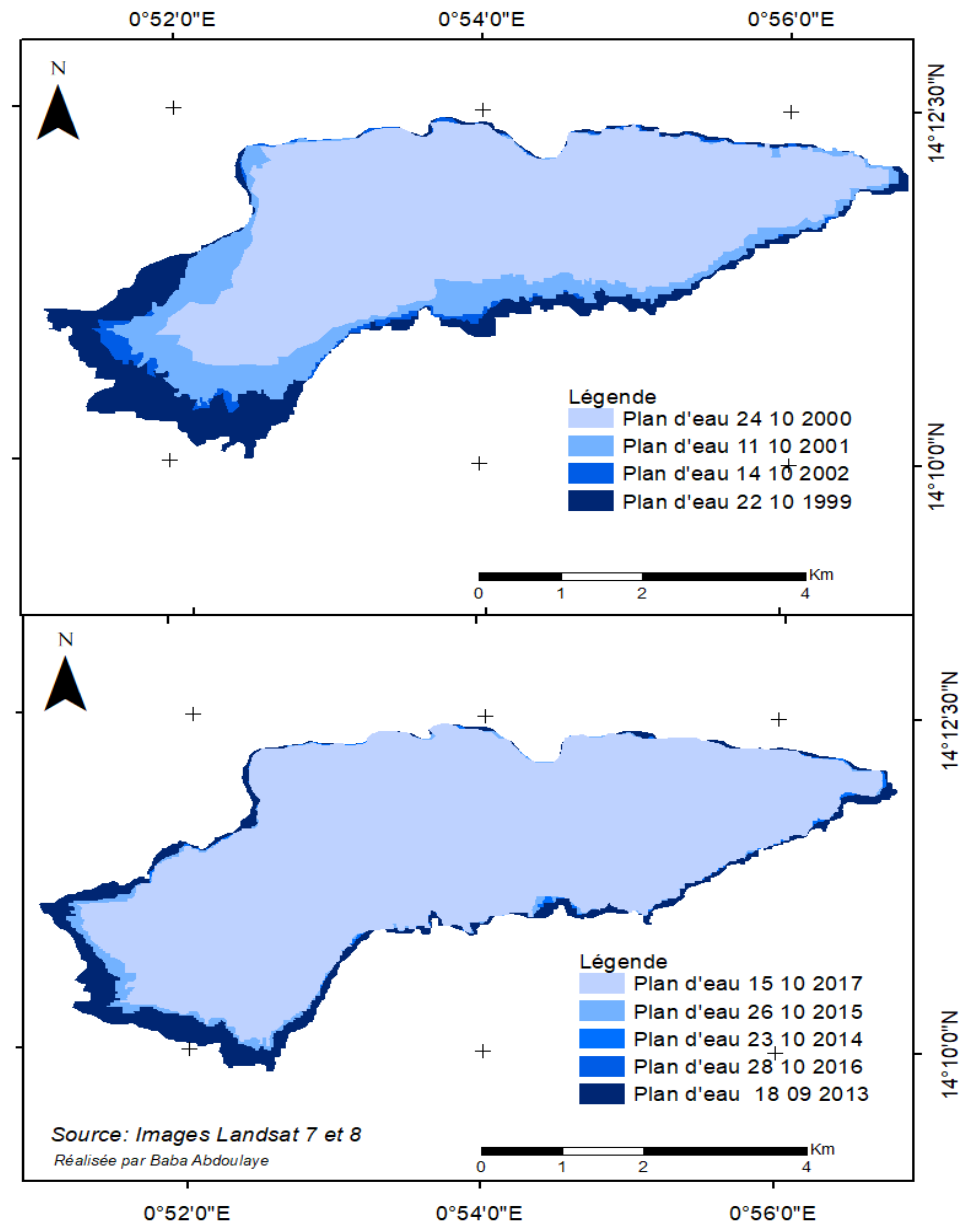

Figure 4 : Variation interannuelle du plan d'eau.

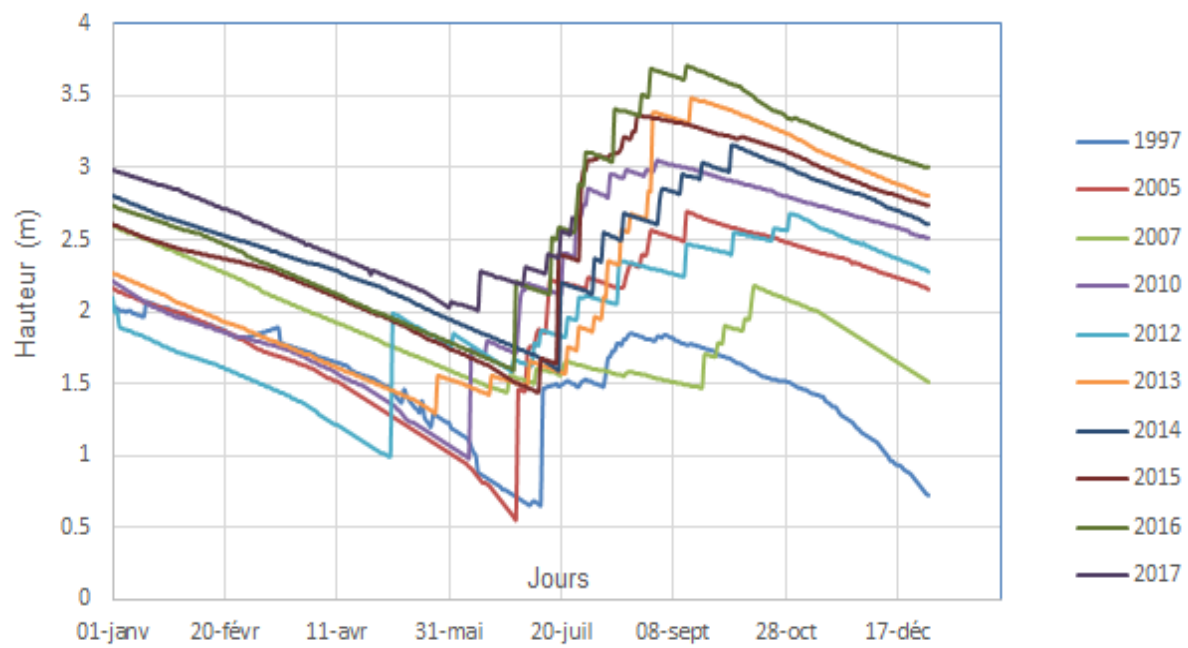

Figure 5 : Variation limnimétrique saisonnière et interannuelle (pour les années régulièrement suivies). Source : (DRRE/TI). 


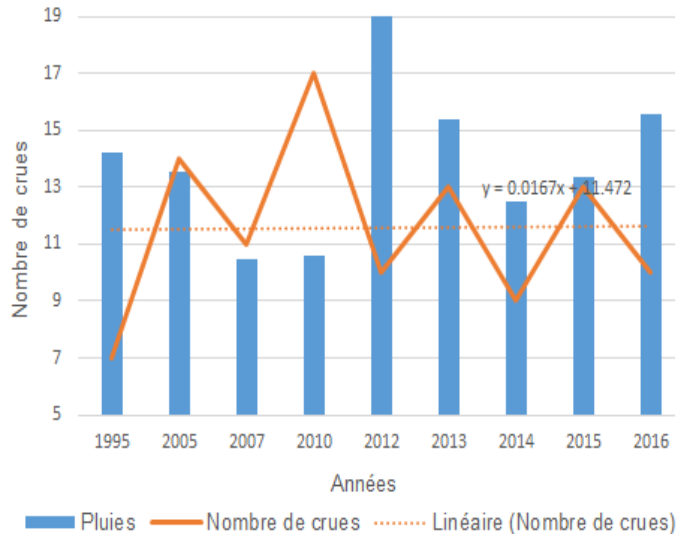

(a)

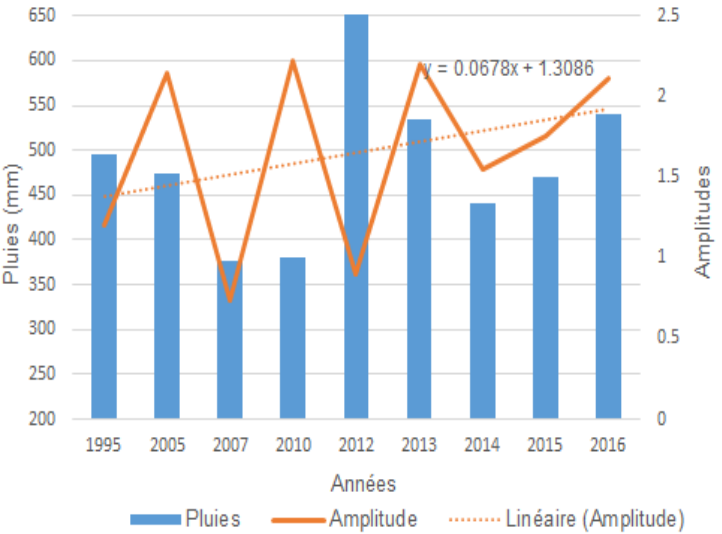

(b)

Figure 6 : Variation du nombre de crues (a) et de de l'amplitude (b) selon les hauteurs pluviométriques pour les années observées. Source : (DRRE/TI).

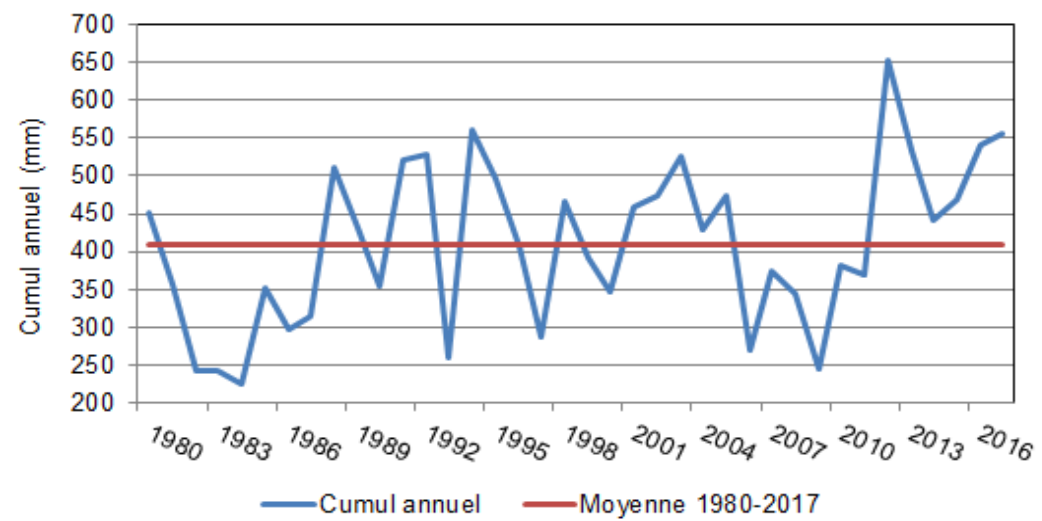

Figure 7: Variation de la pluviométrie de 1980 à 2017 à la station de Téra. Source: DMN.

Tableau 1 : Variation interannuelle des caractéristiques du fonctionnement de la mare.

\begin{tabular}{cccc}
\hline Année & $\begin{array}{c}\text { Date de début } \\
\text { de recharge }\end{array}$ & $\begin{array}{c}\text { Nombre de } \\
\text { crues }\end{array}$ & $\begin{array}{c}\text { Amplitude en fin de } \\
\text { remplissage }(\mathrm{m})\end{array}$ \\
\hline 1997 & $12 / 07 / 1997$ & 9 & 1,20 \\
2005 & $01 / 07 / 2005$ & 15 & 2,16 \\
2007 & $27 / 06 / 2007$ & 11 & 0,74 \\
2010 & $10 / 06 / 2010$ & 18 & 2,22 \\
2012 & $05 / 05 / 2012$ & 10 & 1,68 \\
2013 & $26 / 05 / 2013$ & 13 & 2,2 \\
2014 & $20 / 07 / 2014$ & 9 & 1,55 \\
2015 & $11 / 07 / 2015$ & 14 & 1,91 \\
2016 & $29 / 06 / 2016$ & 10 & 2,11 \\
\hline
\end{tabular}




\section{DISCUSSION}

L'analyse, à l'échelle saisonnière et interrannuelle, des chroniques limnimétriques de la mare de kokorou ainsi que l'exploitation des images Landsat MSS et ETM+ revèlent une forte variabilité des paramètres du fonctionnement de la retenue (Figure 5). La mare de Kokorou présente les caractéristiques d'un milieu sahélien marqué par la forte dépendance aux variations pluviométriques (Figure $6 \mathrm{a}$ et $\mathrm{b}$ ). Ce résultat corrobore ceux obtenus sur le Lac Tchad. En effet, les travaux de Lemoalle (2003) montre une forte sensibilité duLac Tchad aux variabilités climatiques, notamment son bassin nord dont l'inondation a varié d'une année à l'autre et d'une saison à l'autre depuis 1976 avec une étendue annuelle maximale du plan d'eau allant (pour les mois de janvier et février) de zéro (en 1985, 1987 et 1988) à plus de 7000 $\mathrm{km}^{2}$ entre 1976 et 1989 et de 1999 à 2003 . Il corrobore aussi les résultats trouvés sur l'ensemble du plan d'eau du Lac Tchad et sur la partie nigérienne de sa cuvette nord par Lemoalle et al. (2010) repris par Kiari (2014) qui montre une variation du lac de 24000 (superficie proche de celle du "Grand Tchad" , Olivry et al., 1996) à $17000 \mathrm{~km}^{2}$ depuis 1950 et celle de la partie nigérienne de la cuvette nord de 315863 ha en octobre 1963 à 162440 ha 1976. Ces variations des deux bassins du Lac Tchad, conséquemment aux variabilités climatiques, sont également en rapport avec la baisse des apports des affluents du lac à savoir le Chari (pour le bassin sud) et la Komadougou Yobé (pour la partie nigérienne de la cuvette nord). Cependant, sur un autre plan, ces résultats contrastent du fait que la le Lac Tchad est en forte baisse, voire menacé de disparition au moins pour sa partie nigérienne, alors que la mare de Kokorou, elle connaît une extension de sa superficie.

Toujours en zone sédimentaire, le même phénomène est observé au niveau des petites mares et dépressions endoréiques du Continental terminal dans le degré-carré de Niamey. La multiplication du nombre de ces mares au niveau desquelles se concentrent les ruissellements et leur extensionsur des zones non colmatées y est à l'origine de la remontée de la nappe phréatique depuis la séchresse de la fin de la décennie 1960 (Desconnets et al., 1997 ; Leduc et al., 2001 ; Mahé et al., 2003 ; Massuel, 2005 ; Favreau et al., 2009; Ibrahim, et al., 2014).

Dans le Gourma malien, les travaux de Gardelle et al. (2010) montrent également l'extension dede deux mares dont les superficies passent respectivement de 150 à 230 ha et de 300 à 500 ha de 1966 à 2006 et cela, malgré la baisse des précipitations. Il en est de même pour le lac Agoufou dont la superficie est passée de $534 \mathrm{~m}^{2}$ en 1956 à $2.10^{6} \mathrm{~m}^{2}$ en 2011 (Gal, 2016) sous l'effet de la modification des caractéristiques (Gal et al., 2017)

L'accroissement des ruissellements dansle bassin versant de la mare de Kokorou (en particulierdepuis la sécheresse de 2009) à l'origine de la modification de son fonctionnement hydrologique est en concordance avec la situation des ruissellements dans la région de Niamey (Massuel, 2005; Favreau et al., 2009; Ibrahim et al., 2014) et la hausse des écoulements au Sahel (Mahé et al., 2010 ; Descroix et al., 2013; Sighomnou et al., 2013) après les sécheresses des années 19701990. De nombreux travaux ont montré quel'évolution des ruissellments au Sahel découle des changements de l'occupation des sols (Favreau et al., 2009; Bouzou et al., 2011 ; Descroix et al., 2013 ;Malam et al., 2015) et de la remontée récente de la pluviométrie (Massuel, 2005 ; Lebel et Ali, 2009). Nos résultats corroborent cette relation. En effet, comme le confirment $51 \%$ de la population enquêtée, le bassin versant de la mare de Kokorou a connu d'importants changements de l'occupations des sols à l'instar l'ensemble de la zone soudanosahélienne (Avakoudjo et al., 2014 ; Ouédraogo et al., 2017 ; Millogo et al., 2017). Pour la one d'étude, ces changements sont particulièrement importants du faitde l'installation du site orifère de Komabangou en 1984 à $15 \mathrm{~km}$ du bassin versant ce qui a entraîné une modification importante du ruissellement. 
Le résultat obtenu est également en accord avec les modifications pluviométriques en cours dans la zone sahélienne. D'ailleurs, pour le bassin versant de la mare de Kokorou, la situation actuelle constitue un paroxysme dans l'amélioration de la pluviométrie (Figure 7). En effet, la décennie qui commence à partir de 2010 pourrait être la plus pluvieuse avec une moyenne actuelle (2010-2017) de $493 \mathrm{~mm}$ contre une moyenne décennale de $394 \mathrm{~mm}$ entre 2000 et 2009 et $427,5 \mathrm{~mm}$ pour la décennie 1990-1999 qui est déjà considérée comme le début d'une remontée pluviométrique (Ali et Lebel, 2009) au Sahel.

\section{Conclusion}

L'analyse des caractéristiques du fonctionnement de la mare a permis de poser le paradigme du sous la forme d'un modèle de remplissage. La mare de Kokorou, avec jadis un tarissement intervenant en fin de saison sèchequel qu'en soit le niveau de recharge atteint, est actuellement devenue une mare à régime permanent. L'étude a montré une forte croissance de l'intensité de recharge avec un facteur supérieur à 2 en 2008 par rapport à la moyenne des décennies 1990 et 2000, cela malgré la baisse du nombre de crue par phase de recharge. Le fonctionnement actuel est la réponse hydrologique du bassin versant face aux changements intervenus dans l'occupation des sols et la remontée récente de la pluviométrie dans le bassin versant. Ces facteursontinduit un accroissement des apports par ruissellements et par conséquent une extension des superficies de la mare, en fin de recharge, et un accroissement des volumes d'eau stockées.

La présente étude a permis de proposer un modèle de fonctionnement hydrologique pour la mare de Kokorou à partir l'exploitation des données d'archives (Images Landsat MSS, ETM+ et chroniques limnimétriques. La modification de ce fonctionnement depuis la dernière décennie, par accroissement des apports hydriques conséquemment au changement de l'occupation des sols à l'amélioration pluviométrique, est accompagnée de la rupture de l'équilibre du biotope : réduction des ressources halieutiques, disparition quasitotale du fourrage, inondation des terres de cultures, menace d'inondation du village.

\section{CONFLITS D'INTERETS}

Les auteurs déclarent qu'il n'y a aucun conflit d'intérêts pour cet article.

\section{CONTRIBUTION DES AUTEURS}

$\mathrm{AB}$ a assuré la collecte et le traitement des données cartographiques planimétriques et limnimétriques.OFM, OB, DDEC et GF ont participé à la valorisation, à la critique et la validation des données. Ils ont également participé à l'analyse des données et fournit des corrections et commentaires sur la rédaction scientifique de l'article.

\section{REMERCIEMENTS}

La réalisation de cette étude a été rendue possible grâce au concours du Laboratoire Mixte International (LMI PICASS'EAU) qui a pris encharge les missions de terrain d'octobre à décembre 2013. C'est l'occasion de lui adresser nos vifs remerciements.

\section{REFERENCES}

Abdou Babaye MS. 2012. Evaluation des ressources en eau souterraine dans le bassin de Dargol (Liptako-Niger). Thèse de Doctorat, Université de Liège, Belgique, p.244.

Abdou Babaye MS. 2016. Comportement hydrogéochimique et contamination des eaux des aquifères fissurés du socle précambrien en milieu semi-aride (SudOuest du Niger). Int. J. Biol. Chem. Sci., 10(6): 2728-2743. DOI: http://dx.doi.org/10.4314/ijbcs.v10i6.26

Avakoudjo J, Mama A, Toko I, Kindomihou V, Sinsin B. 2014. Dynamique de l'occupation du sol dans le Parc National $\mathrm{du} \mathrm{W}$ et sa périphérie au nord-ouest du Bénin. Int. J. Biol. Chem. Sci., 8(6): 2608-2625.

DOI: http://dx.doi.org/10.4314/ijbcs.v8i6.22

Baba A. 2012. Approche méthodologique pour l'étude du fonctionnement de la mare de Kokorou dans le Liptako 
Gourma (Niger). Mémoire de DEA, Département de Géographie, Université Abdou Moumouni, Niamey, p.77.

Bader JC, Lemoalle J, Leblanc M. 2011. Modèle hydrologique du lac Tchad. Journal des Sciences Hydrologiques, 56(3) : $1-15 . \quad$ DOI : https://doi.org/10.1080/02626667.2011.5 60853

Bouzou MI, Luc D, Faran MO, Gautier E, Mahaman MA, Estèves M, Yéro KS, Malam AM, Mamadou I, Le Breton E,Abba B. 2011. Les changements d'usage des sols et leurs conséquences hydrogéomorphologiques sur un bassin versant endoréique sahélien. Sécheresse, 22(1) : 13-24.

Bahari IM. 2013. Dynamique hydrogéomorphologique actuelle du kori Mountséka Centre-sud, Niger. Thèse de doctorat, Université Abdou Moumouni, Niamey, p.228.

Claude J, Grouzis M, Milleville P. 1991. La Région de la Mare d'Oursi in Un Espace Sahélien, la Mare d'Oursi Burkina Faso.ORSTOM , 23-60.

Desconnets JC, Taupin J D, Lebel T, Leduc C. 1997. Hydrology of the HAPEX-Sahel Central Super-Site: surface water drainage and aquifer recharge through the pool systems. Journal of Hydrology, 188-189(1997) : $155-178 . \quad$ DOI : https://doi.org/10.1016/S00221694(96)03158-7

Descroix L, Bouzou MI, Genthon P, Sighomnou D, Mahé G, Mamadou I, Vandervaere JP, Gautier E, Faran MO, Rajot JL, Mallam AM, Souley YK, Karambiri H, Fensholt R, Albergel J, Olivry JC. 2013. Impact of Drought and Land -Use Changes on Surface -Water Quality and Quantity: The Sahelian Paradox. Intech., 243-271. DOI : https://dx.doi.org/10.5772/54536

Direction Régionale des Ressources en Eau. 2001. Rapport de Suivi de 20 Mares et Retenues Artificielles dans le Liptako Gourma, Novembre 2000-Février 2001 Projet PNUD NER-98/001/NEX. Service des Ressources en Eau. DRRE.
Favreau G, Cappelaere B, Massuel S, Leblanc M, Boucher M, Boulain N, Leduc C. 2009. Land clearing, climate variability, and water resources increase in semiarid southwest Niger : A review. Water Resources Research, 45(7) : 18. DOI: 10.1029/2007WR006785.

Gal L. 2016. Modélisation de l'évolution paradoxale de l'hydrologie sahélienne Application au bassin d'Agoufou (Mali). Thèse de Doctorat, Université Toulouse III Paul Sabatier, Toulouse, p.226.

Gal L. 2017. The paradoxal evolution of runoff in the pastoral Sahel: Analysis of the hydrological changies over the Agoufou watershed (Mali) using the KINOS-2 model. Hydrology and Earth System Sciences, 21: 4591-4613. DOI: https://doi.org/10.5194/hess-21-45912017

Gardelle J, Hiernaux P, Kergoat L, Grippa M. 2010. Less rain, more water in ponds: a remote sensing study of the dynamics surface waters from 1950 to present in pastoral Sahel (Gourma region, Mali). Hydrology and Earth system Sciences, 14: 309-324.

Ibrahim M, Favreau G, Scanlon BR, Seidel JL, Le Coz M, Demarty J, Cappelaere B. 2014. Long-term increase in diffuse groundwater recharge following expansion of rainfed cultivation in the Sahel, West Africa. Hydrogeology Journal, 22: 1293-1305. DOI:10.1007/s10040-014-1143-z.

Kiari F. H. 2014. Impacts des variations du niveau du lac Tchad sur les activités socio-économiques des pêcheurs de la partie nigérienne. Thèse de Doctorat, Université Abdou Moumouni de Niamey, Niger, p.313.

Lebel T, Ali A. 2009. Recent trends in the Central and Western Sahel rainfall regime (1990-2007). Journal of Hydrology, (375): 52-64. DOI : https://doi.org/10.1016/j.jhydrol.2008.11 .030

Leblanc MJ, Favreau G, Massuel S, Tweed S, Loireau M, Cappelaere B. 2008. Land clearance and hydrological change in the 
Sahel : SW Niger. Global and Planetary Change, 61(3-4): 135-150. DOI : https://doi.org/10.1016/j.gloplacha.2007. 08.011

Leduc C, Favreau G, Schroeter P. 2001. Long term-rise in a Sahelian water-table : the Continental Terminal in South-West Niger. Journal of Hydrology, (243): 4354.

Lemoalle J. 2004. Lake Chad: A Changing Environment: In Dying and Dead Seas, Climatic Versus Anthropic Causes. Kluwer Academic Publishers : Amsterdam-Netherlands; 321-340. DOI : 10.1007/978-94-007-0967-6.

Lemoalle J, Bader JC, Leblanc M, Sedick A. 2010. L'évolution récente du lac Tchad: Contexte général et données de base. Ird00579534, Récupéré sur https://hal.archives-ouvertes.fr/ird00579534/document

Mahé G, Diello P, Paturel JE, Barbier B, Karambiri H, Dezetter A, Dieulin, C, Rouché N. 2010. Baisse des pluies et augmentation des écoulements au Sahel : impact climatique et anthropique sur les écoulements du Nakambe au Burkina Faso. Sécheresse, 21(4): 330-332. DOI:10.1684/sec.2010.0279.

Malam MA, Vandervaere JP, Descroix L, Bouzou MI, Faran MO, Abdou Souley, Bodo, S B, Ousseini DML. 2015. Évolution de la conductivité hydraulique d'un sol sableux cultivé du Niger. Biotechnol. Agron. Soc. Environ., 19(3) : 269-279.

Mamadou I, Gautier E, Descroix L, Noma I, Bouzou MI, Faran MO, Genthon P, Amogu O, Mallam AM, Vandervaere JP. 2015. Exorheism growth as an explanation of increasing flooding in the Sahel. Catena, (131) : 130-139. DOI : https://doi.org/10.1016/j.catena.2015.03. 017
Massuel S. 2005. Evolution récente de la ressource en eau consécutive aux changements climatiques et environnementaux du sud-ouest Niger. Modélisation des eaux de surface et souterraines du bassin du kori de Dantiandou sur la période 1992-2003. Thèse de Doctorat, Université Montpellier II, France, p.220.

ME/SU/DD. 2016. Rapport des activités 2015. Rapport Annuel, Maradi, Niger. Consulté le 6 Septembre 2016.

Millogo D, Nikiema A, Koulibaly B, Zombre NP. 2017. Analyse de l'évolution de l'occupation des terres à partir de photographies aériennes de la localité de Loaga dans la province du Bam, Burkina Faso. Int. J. Biol. Chem. Sci., 11(5): 2133-2143. DOI https://dx.doi.org/10.4314/ijbcs.v11i5.16

Olivry JC, Chouret A, Vuillaume G, Lemoalle J, Bricquet J-P. 1996. Hydrologie du lac Tchad. ORSTOM éditions: Paris.

Ouédraogo A, Da ECD, Ouaba PA. 2017. Perception locale de l'évolution du milieu à Oula au Nord du Burkina Faso. Int. J. Biol. Chem. Sci., 11(1) : 144-156. DOI: http://dx.doi.org/10.4314/ijbcs.v11i1.12

Ousmane B, DjiboS, Soumana I,Soussou A. 2010. Etude préliminaire de la pollution bactériologique des eaux des aquifères discontinus du socle du département de Téra/Liptako nigérien. Afrique Science, 3(6) : 27-36. http://www.afriquescience.info

Sighomnou D, Descroix L, Genthon P, Mahé G, Bouzou MI, Gautier E. 2013. La crue de 2012 à Niamey : un paroxysme du paradoxe du Sahel? Science et Changements Planétaires / Sécheresse, 1(24): 3-13. 